\title{
ON THE DIMENSION OF MODULES AND ALGEBRAS, V. DIMENSION OF RESIDUE RINGS
}

\author{
SAMUEL EILENBERG and TADASI NAKAYAMA
}

We shall consider a semi-primary ring $A$ with radical $N$ (i.e. $N$ is nilpotent and $1 / N$ is semi-simple (with minimum condition)). All modules considered are left $\Lambda$-modules. We refer to [1] for all notions relevant to homological algebra.

The objective of this paper is to establish the following two theorems:

THEOREM I. Let a be a two-sided ideal in A such that

$$
a \subset N^{2}, \quad \operatorname{gl} \operatorname{dim}(\Lambda / a) \leqq 1 .
$$

Then $a=0$.

TheOREM II. Let $r$ be a right ideal in A such that

$$
r N \subset N r \subset N^{2}, \quad g l \cdot \operatorname{dim}(\Lambda / N r) \leqq n, \quad n>1 .
$$

Then $N r^{n-1} N=0$.

Taking $r=N^{k-1}, k>1$ we obtain

Corollary II'. If gl.dim $\left(\Lambda / N^{k}\right) \leqq n, k>1, n>1$, then $N^{(n-1)(k-1)+2}=0$.

Corollary II". If $\operatorname{gl} \cdot \operatorname{dim}\left(\Lambda / N^{2}\right) \leqq n, n \geqslant 0$, then $N^{n+1}=0$.

In this last corollary we admitted also the cases $n=0$ ( since $N / N^{2}=0$ implies $N=0$ ) and $n=1$ (by Theorem I). The result stated in Corollary II" is the best possible. Indeed, in [3, Proposition 12 and Corollary 11], for each $n \geqslant 0$, a semi-primary ring $\Lambda$ was constructed such that

$$
\text { gl } \cdot \operatorname{dim} \Lambda \leqq 1, \quad \operatorname{gl} \cdot \operatorname{dim}\left(\Lambda / N^{2}\right)=n, \quad N^{n+1}=0, \quad N^{n} \neq 0 .
$$

Let $\varphi: P \rightarrow A$ be an epimorphism of $A$-modules. We say that $\varphi$ is minimal if $P$ is projective and $\operatorname{Ker} \varphi \subset N P$. We see without much difficulty

(i) For each 1 -module $A$ there is a minimal epimorphism $\varphi: P \rightarrow A$;

Received January 20, 1956. 
(ii) If $\varphi^{\prime}: P^{\prime} \rightarrow A$ is another minimal epimorphism then there exists an isomorphism $\pi: P \rightarrow P^{\prime}$ such that $\varphi^{\prime} \pi=\varphi$;

for the detailed account under a more general setting, see [2].

Let $a$ be any subset of $A$. We define the orthogonality relation $a \perp A$ by the condition $a P=0$, where $P$ is the projective module occuring in the minimal epimorphism for $A$. Clearly $a \perp A$ implies $a A=0$.

Lemma 1. If $B \subset N A$ then the relations $a \perp A$ and $a \perp A / B$ are equivalent.

Lemma 2. If $B \subset N A$ and $A / B$ is projective then $B=0$.

Proof. Consider the composition

$$
P \stackrel{\varphi}{\rightarrow} A \stackrel{\psi}{\rightarrow} A / B
$$

where $\varphi$ is the minimal epimorphism for $A$ and $\psi$ is the natural factorization epimorphism. Since $\operatorname{Ker} \varphi \subset N P$ we have $\varphi^{-1}(B) \subset \varphi^{-1}(N A)=N P$. Thus $\operatorname{Ker}(\phi \varphi) \subset N P$ and $\phi \varphi$ is a minimal epimorphism for $A / B$. Consequently each of the conditions $a \perp A, a \perp A / B$ is equivalent with $a P=0$. If $A / B$ is projective, then, by (ii) $\phi \varphi$ is an isomorphism. Thus $\phi$ is an isomorphism and $B=0$.

Lemma 3. Let $a$ be a two-sided ideal in A, $A$ a A-module and $B$ a submodule such that

$$
a A \subset B \subset N A, \quad A / B \text { is }(A / a) \text {-projective. }
$$

Then $a A=B$.

Proof. Consider the ring $\Lambda^{\prime}=\Lambda / a$ with radical $N^{\prime}=(N+a) / a$. The $\Lambda^{\prime}$-modules $A^{\prime}=A / a A, B^{\prime}=B / a A$ then satisfy

$$
B^{\prime} \subset N^{\prime} A^{\prime}, \quad A^{\prime} / B^{\prime} \text { is } \Lambda^{\prime} \text {-projective. }
$$

Thus, by Lemma $2, B^{\prime}=0$ i.e. $a A=B$.

Proof of Theorem I. Since gl.dim $(\Lambda / a) \leqq 1$ we have $1 \cdot \operatorname{dim}_{\Lambda / a}(\Lambda / N) \leqq 1$. From the exact sequence $0 \rightarrow N / a \rightarrow \Lambda / a \rightarrow \Lambda / N \rightarrow 0$ it follows that $N / a$ is $(\Lambda / a)$. projective. Since $a N \subset a \subset N N$ we may apply Lemma 3 with $(A, B)$ replaced by $(N, a)$. Thus $a N=a$ and $a=0$.

Proposition 4. Let $r$ be a right ideal in $\Lambda$ and $A$ a left $\Lambda$-module. - If

$$
r N \subset N r, \quad r A=0, \quad 1 \cdot \operatorname{dim}_{\Lambda / N r} A \leqq n, \quad n>0
$$

then $N r^{n} \perp A$. 
Proof. Let $\varphi: P \rightarrow A$ be a minimal epimorphism. Since $r A=0$ it follows that $r P \subset \operatorname{Ker} \varphi$. If we write $C=\operatorname{Ker} \varphi$, there results an exact sequence

$$
0 \rightarrow C \rightarrow P \stackrel{\varphi}{\rightarrow} A \rightarrow 0
$$

such that

$$
r P \subset C \subset N P \text {. }
$$

Since $N r P \subset \operatorname{Ker} \varphi$ we derive an exact sequence

$$
0 \rightarrow c / N r P \rightarrow P / N r P \rightarrow A \rightarrow 0
$$

of $(\Lambda / N r)$-modules. Since $P / N r P$ is $(\Lambda / N r)$-projective (see [3, Prop. 1]), we have

$$
\operatorname{lidim}_{\Lambda / N r}(C / N r P) \leqq n-1 \text {. }
$$

Now consider the case $n=1$. Since

$$
N r C \subset N r P \subset N C
$$

we may apply Lemma 3 with $(A, B, a)$ replaced by $(C, N r P, N r)$. We obtain $N r C=N r P$. Since $C \subset N P$ and $r N \subset N r$ we have

$$
N r P=N r C \subset N r N P \subset N^{2} r P .
$$

Thus $N r P=0$ i.e. $N r \perp A$.

For $n>1$ we proceed by induction and assume the proposition valid for $n-1$. Since $r C \subset r N P \subset N r P$ we have $r(C / N r P)=0$ and thus $(*)$ yields

$$
N r^{n-1} \perp C / N r P \text {. }
$$

However $N r P \subset N C$, so that, by Lemma $1, N r^{n-1} \perp C$. Consequently $N r^{n} P \subset$ $N r^{n-1} C=0$ and $N r^{n} \perp A$.

Proof of Theorem II. Since gl.dim $(\Lambda / N r) \leqq n$ we have $\operatorname{ldim}_{\Lambda / N r}(\Lambda / N) \leqq n$. From the exact sequence $0 \rightarrow N / N r \rightarrow \Lambda / N r \rightarrow \Lambda / N \rightarrow 0$ it follows

$$
\operatorname{lidim}_{\Lambda / N r}(N / N r) \leqq n-1 .
$$

Since $r N \subset N r$ we have $r(N / N r)=0$ and thus, by Proposition 4,

$$
N r^{n-1} \perp N / N r .
$$

Since $N r \subset N N$, it follows from Lemma 1 that 


$$
N r^{n-1} \perp N
$$

Thus $N r^{n-1} N=0$, as required.

\section{REFERENCES}

[1] H. Cartan and S. Eilenberg, Homological Algebra, Princeton Univ. Press, 1956.

[2] S. Eilenberg, Homological dimension and syzygies, Ann. Math., 64 (1956), 328-336.

[3] S. Eilenberg, H. Nagao and T. Nakayama, On the dimension of modules and algebras, IV, Nagoya Math. J., 10 (1956), 87-95.

Columbia University

Nagoya University and The Institute for Advanced Study 